\title{
English-learning infants' perception of word stress patterns
}

\author{
Katrin Skoruppa \\ Department of Speech, Hearing and Phonetic Sciences, University College London, \\ London WC1N 1PF, United Kingdom \\ kskoruppa@gmail.com \\ Alejandrina Cristiàa) and Sharon Peperkamp \\ Laboratoire de Sciences Cognitives et Psycholinguistique, EHESS, DEC-ENS, \\ CNRS, 75005 Paris, France \\ alecristia@gmail.com, Sharon.Peperkamp@ens.fr \\ Amanda Seidl \\ Department of Speech, Language, and Hearing Sciences, Purdue University, \\ West Lafayette, Indiana 47907 \\ aseidl@purdue.edu
}

\begin{abstract}
Adult speakers of different free stress languages (e.g., English, Spanish) differ both in their sensitivity to lexical stress and in their processing of suprasegmental and vowel quality cues to stress. In a head-turn preference experiment with a familiarization phase, both 8month-old and 12-month-old English-learning infants discriminated between initial stress and final stress among lists of Spanish-spoken disyllabic nonwords that were segmentally varied (e.g. ['nila, 'tuli] vs [lu'ta, pu'ki]). This is evidence that English-learning infants are sensitive to lexical stress patterns, instantiated primarily by suprasegmental cues, during the second half of the first year of life.
\end{abstract}

(C) 2011 Acoustical Society of America

PACS numbers: 43.71.Ft, 43.71. Hw [JH]

Date Received: March 10, $2011 \quad$ Date Accepted: April 13, 2011

\section{Introduction}

Languages differ with respect to the lexical stress patterns they permit. In some languages, such as English and Spanish, word stress can vary freely and convey lexical distinctions (e.g., English: discount ['diskaunt] vs discount [dis'kaunt]; Spanish: sábana ['saßana] - 'sheet' vs sabana [sa'ßana] - 'savannah'). In other languages, stress is fixed and always falls on the same syllable position within words, e.g., Hungarian words are stressed on the first syllable, Swahili words on the penultimate. These cross-linguistic differences impact adult speakers' stress perception abilities: Speakers of French, Finnish, Hungarian, and Polish, all fixed stress languages, are less proficient than Spanish speakers at distinguishing nonwords that differ only in their stress pattern (e.g., ['mapi] vs [ma'pi]) in a sequence recall task (Peperkamp et al., 2010). In contrast, speakers of English, a free stress language, are able to perform a range of phonological and lexical tasks based on stress: They can identify stressed syllables based on suprasegmental cues alone (Fry, 1958) and integrate stress cues in cross-modal lexical priming (Cooper et al., 2002).

However, free stress languages are not a homogenous group. First, there are differences in the distribution of stress patterns, with many free stress languages being less free than it would appear at first glance. For example, roughly $60 \%$ of the

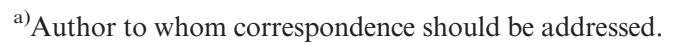


disyllabic words have initial stress in the Spanish lexicon, compared to $75 \%$ of disyllabic words in the English lexicon (Pons and Bosch, 2010). Second, languages also differ with respect to the acoustic realization of lexical stress. Most, including Spanish, use some combination of suprasegmental cues (most importantly, pitch, duration, and amplitude) (Fry, 1958). Other languages, including English, further recruit segmental cues, by introducing important changes in vowel quality in unstressed syllables (Fry, 1958). Although vowels of shorter duration are centralized due to undershoot in many languages, this phenomenon is greatly amplified for unstressed vowels in English, where vowel reduction is five times larger than in Spanish (Delattre, 1969).

Given that free stress languages are not a homogenous group, it is not surprising that stress perception abilities of speakers of these languages vary as well, and may be modulated by the distributional and acoustic differences sketched previously. For instance, Spanish listeners' reaction times are always negatively affected by stress mismatches in a cross-modal priming task (Soto-Faraco et al., 2001), whereas English listeners only exhibit slowed responses in short words (Cooper et al., 2002). In fact, suprasegmental differences alone, i.e., the differences between minimal pairs that do not differ in vowel quality, e.g., forebear ['fobeə] vs forbear [fo'beə] - do not disrupt priming in British English listeners (Cutler, 1986). Further, stress misplacement only affects word recognition if it results in a nonword (Small et al., 1988): for instance, polite produced with incorrect initial stress inhibits word recognition, whereas the noun insert produced with incorrect final stress does not. Finally, Fear et al. (1995) report that English listeners are more sensitive to changes in vowel quality than to changes in suprasegmental stress cues. To sum up, as stress is more regular and relies on more diverse cues in English than in Spanish, English listeners are overall less sensitive to stress, and when they do attend to it, they seem to give less weight to suprasegmental cues than Spanish listeners.

Cross-linguistic differences in infants' stress sensitivity in words with varying vowels and consonants have only recently begun to be investigated (see Skoruppa et al., 2009, for a comprehensive review of infant stress perception research with segmentally nonvaried stimuli). The perception of stress patterns in segmentally varied stimuli differs between infants learning fixed and free stress languages already at the age of 9 months. At this age, Spanish-learning infants discriminate stress patterns (such as ['lapi 'naku] vs [ki'bu lu'ta]), whereas French-learning infants do not discriminate stress patterns in variable stimuli, although they can distinguish stress patterns in repeated identical nonwords (['pima] vs [pi'ma]) (Skoruppa et al., 2009).

Cross-linguistic differences have also been found among learners of free stress languages. English learners prefer stress-initial over stress-final disyllabic real words (e.g., pliant, falter vs comply, befall) at 9 but not at 6 months of age (Jusczyk et al., 1993). Turk et al. (1995) found a similar preference in English-learning 9-month-olds using nonwords (e.g., ['.Jezəl 'zi:ləl] vs [lə'.ıez lə'zi:1]). Interestingly, Spanish-learning 9month-olds show no overall preference for either of these patterns (e.g. ['kiba 'buki] vs [ni'ka bi'lu]) (Pons and Bosch, 2010). This difference could indicate that even children learning different free stress languages begin to show diverse perceptual patterns in the first year of life, similar to those found in adults. Indeed, the preference in Englishlearning, but not Spanish-learning, infants could be explained on the basis of differences in overall frequency (as stress-initial disyllables occur more frequently in English than in Spanish). Evidence that stress-initial words have a special status for Englishlearning infants also comes from a study on early word segmentation: Jusczyk et al. (1999) report that 7.5-month-olds can extract unfamiliar stress-initial words such as hamlet ['hæmlət] from continuous speech, but they missegment stress-final words such as device [di'vais] until the age of 10.5 months. A similar preference for initial stress has also been found in an artificial language learning study using longer stimuli (Gerken, 2004): Tested on nonwords with five syllables, 9-month-old American infants showed an overall preference for English-like stress on the first and fourth syllables (e.g., dóremitónfa) over stress on the second and fifth syllables (e.g., dotónremifá), 
despite the fact that half of them had been familiarized with stimuli following the latter pattern. However, infants were able to learn other novel rules involving stress and heavy syllables in this study, suggesting that their stress perception abilities are not entirely rigid. Taken together, these studies suggest that lexical stress perception may be more flexible and less biased toward initial stress in Spanish- than in English-learning infants.

Finally, the weighting of suprasegmental and segmental cues to stress may also develop differently depending on language exposure. In particular, English-learning infants, like English-speaking adults (Fear et al., 1995) may rely more heavily on vowel reduction. As a matter of fact, Jusczyk et al. (1993; Exp. 3) report that 9month-old English-learning infants' preference for stress-initial words holds even when stimuli are low-pass filtered. However, this only demonstrates that English-learning infants are sensitive to suprasegmental cues in the absence of segmental content; it does not show whether they can rely mainly on suprasegmental cues when segmental content is available. Evidence that prosodic information may be harder to attend to in the presence of segmentally varied content comes from a study on pitch perception (Lebedeva and Kuhl, 2010), which shows that 11-month-old American infants can detect melody inversions if the pitch changes are presented on four identical syllables (i.e., lalalala), but not if they are presented on four different syllables (i.e., gobiratu).

Thus, the present study set out to investigate whether English-learning infants can encode lexical stress patterns both in stress-initial and in stress-final words, based principally on suprasegmental cues. In order to have an established point of comparison, we used the same (Spanish) stimuli and method (head-turn preference procedure) as in Skoruppa et al. (2009). This also allowed us to address the question of whether infants can discriminate between stress patterns in foreign words, despite the fact that the phonetic realization of both the segments and the stress cues are unfamiliar to them. Considering previous evidence of a shift in stress-related segmentation abilities between 7.5 and 10.5 months in English-learning infants (Juszyk et al. 1999), we tested infants at two age ranges within the second half of the first year of life.

\section{Method}

\subsection{Participants}

Fifty-six healthy full-term English-learning infants ( 27 girls, 29 boys) were tested in West Lafayette, IN. Half of them were around eight months (mean 7;30, range 7;17-8;24) and half of them around 12 months old (mean 11;30, range 11;07-12;20). A further 15 infants participated whose results are not reported for the following reasons: 14 for fussing or crying; and 1 for having a total looking time of less than $1 \mathrm{~s}$ for one test trial type.

\subsection{Materials}

The stimuli, listed in Table 1, were the same as in Skoruppa et al. (2009). They had been produced in infant-directed speech by a female native-speaker of Spanish. There were $16 \mathrm{CVCV}$ nonwords. Eight nonwords with initial stress and eight segmentally

Table 1. Stimuli.

\begin{tabular}{|c|c|c|c|c|c|}
\hline \multicolumn{4}{|c|}{ Familiarization } & \multirow{2}{*}{\multicolumn{2}{|c|}{$\frac{\text { Test }}{\text { All infants }}$}} \\
\hline \multicolumn{2}{|c|}{ Stress-initial group } & \multicolumn{2}{|c|}{ Stress-final group } & & \\
\hline List 1 & List 2 & List 1 & List 2 & Stress-initial list & Stress-final list \\
\hline 'datu & 'latu & da'tu & la'tu & 'lapi & ki'ßu \\
\hline 'sapi & 'buki & sa'pi & bu'ki & 'naku & lu'ta \\
\hline 'kißa & 'luma & ki'ßa & lu'ma & 'nila & pi'ma \\
\hline 'nuki & 'tiku & nu'ki & ti'ku & 'tuli & pu'ki \\
\hline
\end{tabular}


identical nonwords with final stress were used for familiarization. Eight different nonwords, four with initial stress and four with final stress, were used for the test phase. Acoustic measurements revealed that stress was instantiated by significant differences in duration, intensity, and pitch between stressed and unstressed vowels (all $p$ 's $<0.001$ ); further details can be found in Skoruppa et al. (2009).

\subsection{Procedure}

A variant of the head-turn preference procedure (Jusczyk and Aslin, 1995) was used. Infants were tested in a sound-attenuated, dimly lit room, while seated on a caregiver's lap in the middle of a three-walled enclosure. Behind three openings in the front panel of the enclosure, out of sight of the infant, was located an experimenter who observed the infant's head-turns through a camera, and recorded them using a response box. Both the caregiver and the experimenter listened to music designed to mask human speech over Peltor Aviation headphones. Approximately at eye level for the infant, there was a green light on the front panel and a red light on each side panel, and behind the latter there were speakers. Each trial started with a green light flashing on the front panel. As soon as the infant fixated on it, it was extinguished, and one of the red side lights began to flash. Once the infant oriented toward the side light, the stimulus presentation began, and continued until the infant turned away for more than $2 \mathrm{~s}$ or until the stimulus list had been repeated three times. The time spent oriented toward the source of the sound ("looking time") is the dependent measure, a proxy for infants' attention.

Infants were randomly assigned to the "stress-initial" or "stress-final" group. During familiarization, infants in the stress-initial group heard the two stress-initial familiarization lists; similarly, the stress-final group heard the two stress-final familiarization lists (see Table 1). The interstimulus interval coherence was fixed at $1.8 \mathrm{~s}$. The side of the light and the list being played, alternated until the infant had accumulated $1 \mathrm{~min}$ of total attention time for each list. The subsequent four-trial test phase was identical for all infants. There were two trials with a list of new stress-initial nonwords and another two with a list of new stress-final nonwords (see Table 1). The order and side of presentation of the two lists were randomized.

\section{Results}

Mean looking times for familiar vs novel stress patterns by age and familiarization group are shown in Table 2. A repeated measures analysis of variance with the withinsubject factor Stress Pattern (familiar vs novel) and the between-subject factors Age (8 months vs 12 months) and Familiarization (stress-initial vs stress-final) revealed a significant main effect of Stress Pattern: Looking times were significantly higher for the novel than for the familiar stress pattern $[F(1,52)=11.70, p<0.001]$. There was also a marginal main effect of Familiarization: Infants familiarized with stress-initial nonwords had marginally higher looking times than infants familiarized with stress-final nonwords $[F(1,52)=3.80, p=0.057]$. All other effects and interactions were not significant $(F<1)$, showing, in particular, that there were no age-related changes. Nonparametric analyses using a Pearson's $\chi^{2}$ test confirmed the main result: 40 infants (out of 56) showed longer looking times to the novel stress pattern $\left[\chi^{2}(1)=10.26, p=0.001\right]$.

Table 2. Mean looking times (standard deviation) in seconds.

\begin{tabular}{|c|c|c|c|c|c|c|}
\hline \multirow{2}{*}{$\begin{array}{l}\text { Familiarization } \\
\text { Age }\end{array}$} & \multicolumn{2}{|c|}{ All } & \multicolumn{2}{|c|}{ Stress-initial } & \multicolumn{2}{|c|}{ Stress-final } \\
\hline & Familiar & Novel & Familiar & Novel & Familiar & Novel \\
\hline All & $6.75(4.56)$ & $9.94(6.33)$ & $7.84(5.23)$ & $11.09(6.75)$ & $5.66(3.55)$ & $8.80(5.77)$ \\
\hline 8 months & $7.12(4.63)$ & $10.40(6.92)$ & $8.49(4.91)$ & $11.65(7.85)$ & $5.75(4.04)$ & $9.16(5.88)$ \\
\hline 12 months & $6.38(4.55)$ & $9.48(5.77)$ & $7.18(5.63)$ & $10.53(5.70)$ & $5.57(3.14)$ & $8.44(5.86)$ \\
\hline
\end{tabular}




\section{Discussion}

After 2 min of familiarization with disyllabic nonwords that shared the same stress pattern (stress-initial or stress-final), both 8- and 12-month-old English-learning infants listened longer to novel nonwords with the opposite stress pattern. As test and familiarization stimuli differed in their segmental content, focusing on segmental differences or recalling particular tokens could not help infants in differentiating between the two test lists. Therefore, the novelty preference observed at both ages suggests that, like Spanish learners and unlike French ones (Skoruppa et al. 2009), infants learning English are able to discriminate stress patterns in segmentally varied nonwords. This is all the more remarkable given that the consonants and vowels in the stimuli were produced by a foreign language speaker who used different stress cues as well as language-specific realizations of vowels and consonants.

Skoruppa et al. (2009) showed that the fundamental distinction between speakers of fixed stress languages and speakers of free stress languages (Peperkamp et al., 2010) is present from the first year of life. Given that adult research also documents variation in perception among free stress languages, one might expect English infants' performance with Spanish stimuli to be intermediate between that of Spanish and French learners. This would be a precursor of the reduced lexical stress sensitivity recorded in adults, particularly when vowel quality is relatively preserved (e.g., SotoFaraco et al., 2001 vs Cooper et al., 2002). This prediction was not met, lending little support to the hypothesis that differences in the distribution and realization of stress patterns in the Spanish and English input would affect Spanish and English infants' sensitivity to stress very early on, at least not at the ages we tested and with the task and stimuli we used. Of course, a more sensitive procedure might reveal possible differences between Spanish and English infants; for instance, it would be interesting to use electrophysiological measurements of auditory evoked potentials, which can reveal a more graded response pattern at the neural level.

This is not to say that cross-linguistic differences in lexical stress have no impact in infancy. On the contrary, as noted in the Introduction, previous work documents differences in infants' preferences (Turk et al., 1995 vs Pons and Bosch, 2010). These were (partly) replicated here, as we found a marginal trend for greater overall looking times in infants familiarized with stress-initial nonwords. However, the lack of an interaction between familiarization group and trial type at test suggests that these preferences did not impede infants' discrimination of stress patterns. Taken together with previous research, our results suggest that while differences in frequency of occurrence and realization of stress patterns affect prelinguistic infants' preferences, these differences do not, in their stead, block the representation of stress in segmentally variable material. More generally, the present work suggests that differences in sensitivity to lexical stress across various types of free stress languages that have been documented in adult listeners cannot yet be demonstrated during the first year of life.

\section{Acknowledgments}

We thank Laura Bosch and Begoña Diaz for providing the stimuli, Ashley Foxworthy, Amanda Schultz, Carrie Wade, and Yuanyuan Wang for help with recruiting and testing, and all babies and parents for their participation. The first and the second author contributed equally to this study.

\section{References and links}

Cooper, N., Cutler, A., and Wales, R. (2002). "Constraints of lexical stress on lexical access in English: Evidence from native and non-native listeners," Lang. Speech 45, 207-228.

Cutler, A. (1986). "Forbear is a homophone: Lexical prosody does not constrain lexical access," Lang. Speech 29, 201-220.

Delattre, P. (1969). "An acoustic and articulatory study of vowel reduction in four languages," Int. Rev. Appl. Ling. Lang. Teach. 7, 295-325. 
Fear, B. D., Cutler, A., and Butterfield, S. (1995). "The strong/weak syllable distinction in English,” J. Acoust. Soc. Am. 97, 1893-1904.

Fry, D. B. (1958). "Experiments in the perception of stress," Lang. Speech, 1, 126-152.

Gerken, L.A. (2004). "Nine-month-olds extract structural principles required for natural language," Cognition 93, B89-B96.

Jusczyk, P. W., and Aslin, R. N. (1995). "Infants' detection of the sound patterns of words in fluent speech,” Cognit. Psychol. 29, 1-23.

Jusczyk, P. W., Cutler, A., and Redanz, N. (1993). "Infants' preference for the predominant stress patterns of English words," Child Dev. 64, 675-687.

Jusczyk, P. W., Houston, D. M., and Newsome, M. (1999). "The beginnings of word segmentation in English-learning infants," Cognit. Psychol. 39, 159-207.

Lebedeva, G. C., and Kuhl, P. K. (2010). "Sing that tune: Infants' perception of melody and lyrics and the facilitation of phonetic recognition in songs," Infant Behav. Devel. 33, 419-430.

Peperkamp, S., Vendelin, I., and Dupoux, E. (2010). "Perception of predictable stress: A cross-linguistic investigation," J. Phonet. 38, 422-430.

Pons, F., and Bosch, L. (2010). "Stress pattern preference in Spanish-learning infants: The role of syllable weight," Infancy 15, 223-245.

Skoruppa, K., Pons, F., Christophe, A., Bosch, L., Dupoux, E., Sebastián-Gallés, N., Alves Limissuri, R., and Peperkamp, S. (2009). "Language-specific stress perception by 9-month-old French and Spanish infants," Dev. Sci. 12, 914-919.

Small, L. H., Simon, S. D., and Goldberg, J. S. (1988). "Lexical stress and lexical access - homographs versus nonhomographs," Percept. Psychophys. 44, 272-280.

Soto-Faraco, S., Sebastián-Gallés, N., and Cutler, A. (2001). "Segmental and suprasegmental mismatch in lexical access," J. Mem. Lang. 45, 412-432.

Turk, A. E., Jusczyk, P. W., and Gerken, L. A. (1995). "Do English- learning infants use syllable weight to determine stress," Lang. Speech 38, 143-158. 